\title{
EFEITOS PSICOLÓGICOS DO ISOLAMENTO SOCIAL NO BRASIL DURANTE A PANDEMIA DE COVID-19
}

\section{ARTIGO DE REVISÃO}

MAGALHÃES, Ricardo Antonio ${ }^{1}$

GARCIA, July Mesquita Mendes ${ }^{2}$

MAGALHÃES, Ricardo Antonio. GARCIA, July Mesquita Mendes. Efeitos Psicológicos do Isolamento Social no Brasil durante a pandemia de COVID-19. Revista Científica Multidisciplinar Núcleo do Conhecimento. Ano 05, Ed. 01, Vol. 01, pp. 18-33. Janeiro de 2021. ISSN: 2448-0959, Link de acesso: https://www.nucleodoconhecimento.com.br/psicologia/isolamento-social

\section{RESUMO}

Neste estudo foram levantados os impactos da Covid-19 na saúde mental. Especificamente as doenças mentais observadas ao longo do isolamento social ocasionado pela pandemia. $\mathrm{O}$ estudo almejou responder à seguinte questão: quais os efeitos psicológicos observados na literatura atual sobre o isolamento social causado pela COVID-19? Quanto a natureza esta pesquisa se caracteriza como aplicada, em relação a sua forma de abordagem como qualitativa, no que diz respeito aos seus objetivos a pesquisa é classificada como descritiva e quanto aos procedimentos técnicos como levantamento. Como resultados, observou-se que a Covid-19 proporciona alterações na saúde mental da população com aumento do número de sintomas de Transtorno de Estresse Pós-Traumático, transtornos de ansiedade e afetos negativos após o final do isolamento social. O distanciamento físico reduziu a

1 Graduando em Psicologia na Universidade Estácio de Sá, e Graduado em Tecnologia em Segurança do Trabalho pelo Centro Universitário Gama e Souza.

${ }^{2}$ Graduanda em Psicologia na Universidade Estácio de Sá, e graduada em Gestão de Recursos Humanos pela Universidade Estácio de Sá. 
disponibilidade de muitos apoios familiares, sociais e psiquiátricos. Pessoas com doenças mentais graves e desvantagens socioeconômicas associadas estão particularmente sob risco dos efeitos diretos e indiretos da pandemia. No entanto, conclui-se que a COVID-19 não só causa problemas de saúde física, mas também resulta em uma série de distúrbios psicológicos. A disseminação da COVID-19 pode impactar a saúde mental de pessoas em diferentes comunidades.

Palavras-chave: Covid-19, isolamento social, saúde mental.

\section{INTRODUÇÃO}

Em dezembro de 2019 a China informou à Organização Mundial da Saúde (OMS) sobre um surto de uma nova doença, semelhante a uma pneumonia. Essa doença, transmitida pelo novo Coronavírus, posteriormente identificado como o Sars-Cov-2, foi denominada COVID-19. Em janeiro de 2020, novos casos da COVID-19 foram notificados fora da China, o que levou a OMS declarar emergência internacional em saúde pública. Na América Latina, o primeiro caso registrado foi em São Paulo, no Brasil, no dia 25 de fevereiro de 2020. De acordo com o relatório de situação 115 , emitido pela OMS, até 14 de maio de 2020 foram confirmados 4.248 .389 de casos e 292.046 mortes pela doença em todo mundo. No Brasil, na mesma data, foram confirmados 177.589 casos e 12.400 mortes, de acordo com o boletim diário da OMS (WHO, 2020). Nesta situação, o status da doença se modificou, pela alta taxa de transmissão do vírus e sua propagação em nível mundial, passando à classificação de pandemia, segundo relatório de situação no 114, emitido pela OMS.

Mais de 180 países ao redor do mundo registraram a presença do COVID-19, e mediante a disseminação deste vírus, várias autoridades governamentais têm adotado diversas estratégias, objetivando a redução do ritmo da sua progressão (KRAEMER et al., 2020). Sendo identificada como uma doença predominantemente respiratória, a COVID-19 pode ser transmitida de pessoa para pessoa através de gotículas de saliva, espirro, tosse e catarro, bem como pelo contato próximo a boca, nariz, olhos, ou objetos e superfícies contaminadas (TEIXEIRA, 2020). 
A situação em rápida evolução alterou drasticamente a vida das pessoas, bem como vários aspectos da economia global, pública e privada. Declínios no turismo, aviação, agricultura e na indústria de finanças, devido a pandemia de COVID-19, são relatados como reduções massivas em ambos os aspectos de oferta e demanda da economia que se tornaram consequências inevitáveis e que dependeram de adoção de estratégias por governos internacionalmente.

Dentre as estratégias necessárias, verifica-se que o distanciamento social foi a primeira medida de prevenção adotada com o propósito de evitar aglomerações, a fim de manter, no mínimo, um metro e meio de distância entre as pessoas, assim como a proibição de eventos que ocasionem a reunião de um grande número de indivíduos (escolas, universidades, shows, shoppings, academias esportivas, eventos esportivos, entre outros) (REIS et al., 2020).

Nessa perspectiva, observa-se que juntamente com a pandemia de COVID-19 surge um estado de pânico social em nível global e a sensação do isolamento social desencadeia os sentimentos (de angústia, insegurança e medo), que podem se estender até mesmo após o controle do vírus (HOSSAIN et al., 2020).

Nesse sentido, apesar de o isolamento social ser uma medida bastante empregada no âmbito de saúde pública visando a preservação da saúde física do indivíduo, é fundamental pensar na saúde mental e no bem estar das pessoas submetidas a esse período de isolamento social. Ressaltando tal fato, este estudo objetivou realizar uma análise quanto as consequências ocasionadas na saúde mental, sucedidas do período de isolamento social durante a pandemia da COVID-19, pronunciando, ao fim , estratégias de enfrentamento para minimizá-las.

No Brasil, verifica-se que a grande desigualdade social e regional tem dificultado o processo de isolamento, visto que a população de pessoas pobres e extremamente pobres, que compõem mais de 66 milhões brasileiros, necessita sair de casa em busca da sua subsistência e a da sua família, aumentando, assim, o risco de infecção e transmissão do novo Coronavírus (OLIVEIRA et al., 2020). 
Neste contexto, a praticabilidade do isolamento social durante a pandemia resulta na exposição do sofrimento de uma grande parte da população, causados pelas exacerbadas preocupações como: problemas ligados a economia e a explosão de notícias, bem como outras questões relacionadas a realidade de cada uma das pessoas que mantem relações no cotidiano. Fatores como esses podem ocasionar a manifestação ou o agravamento de sintomas psicológicos como a ansiedade, o estresse e a depressão (OrRNELL et al., 2020). Vale ressaltar que não existe apenas um grupo específico que venha sofrer com os efeitos do isolamento social, assim, todas as pessoas estão sujeitos aos efeitos psicológicos ocasionados durante esse período de pandemia, independentemente de suas idades ou grupos sociais (MARTINS et al., 2020).

As incertezas e os medos associados à pandemia do Coronavírus, juntamente com os bloqueios em massa e a recessão econômica se demostraram com potencial para elevar a incidência do suicídio, bem como dos transtornos mentais associados ao suicídio (MAVROUDEAS, 2020).

Em diferentes níveis o processo de isolamento social tem causado muitos impactos na vida das pessoas. Neste sentido, esta pesquisa buscou compreender os principais efeitos do isolamento social, analisando os fatores que cooperam ou atrapalham esse processo, correlacionando também algumas características socioeconômicas da população com os fatores relacionados ao isolamento social.

A proposta deste artigo tende ao campo da Psicologia e trata sobre a perspectiva da produção de materiais informativos direcionados a saúde mental da população presente num cenário bastante delicado quanto ao enfrentamento da COVID-19. Falar a respeito da saúde mental em períodos de pandemia é crucial, em razão do afastamento e/ou isolamento social estar presente dentre as medidas preventivas, sendo esta considerada uma das mais impactantes, visto que pode levar as pessoas a desenvolverem diversos transtornos emocionais, tais como o estresse, a ansiedade, o pânico entre outros (RIBEIRO, 2020). 
Para a obtenção dos resultados, optou-se pelo método de revisão sistemática de literatura com abordagem qualitativa e de caráter exploratório, em que os autores tiveram como objetivo: compreender melhor os impactos no cotidiano da população frente ao isolamento social por Coronavírus, tornando-o mais explícito.

\section{METODOLOGIA}

Quanto a natureza, esta pesquisa se caracteriza como aplicada, em relação a sua forma de abordagem como qualitativa, no que diz respeito aos seus objetivos a pesquisa é classificada como descritiva e quanto aos procedimentos técnicos como levantamento.

A coleta de dados foi processada a partir de levantamento bibliográfico por consulta, realizada pelo acesso as plataformas digitais como Biblioteca Virtual da Estácio, Portal de Periódicos EBSCO-Estácio, Periódicos DynaMed, Meu Pergamum e acervo, SCIELO, PubMed, Lilacs, Bireme, Google acadêmico e Periódico Capes.

Foram utilizados como descritores, a fim de se delimitar a pesquisa, as palavras: "COVID-19", "pandemia", saúde mental", "doenças mentais", "isolamento social". Foram considerados publicações em Língua Portuguesa, Língua Inglesa e Língua espanhola, publicados em revistas indexadas do ano 2004 até 2020.

Foi adotado como critério de inclusão a admissão de bibliografias que respondessem à pergunta norteadora ligada ao objetivo geral deste artigo: Quais os efeitos psicológicos observados na literatura atual sobre o isolamento social causado pela COVID-19? Em seguida, procedeu-se a leitura dos resumos das publicações e foram excluídas as que não se enquadravam em critérios como, por exemplo, a menção de casos sobre território brasileiro, ou a apresentação de características culturais diferentes da realidade brasileira.

\section{RESULTADOS}

Das 110 publicações levantadas com o emprego dos descritores, 60 foram descartadas por não se enquadrarem nos critérios de inclusão, finalmente, a última 
etapa referente a leitura integral das 50 publicações restantes, possibilitou na utilização de 38 para a produção final deste artigo e que se encaixaram nos critérios de inclusão e exclusão.

Tabela 1 - Seleção das publicações por tema, autores e ano.

\begin{tabular}{|c|c|c|}
\hline Tema & Autores & Ano \\
\hline Efeitos psicológicos do isolamento social & $\begin{array}{l}\text { Brooks et al.; } \\
\text { Zanon et al.; } \\
\text { JOHAL. }\end{array}$ & $\begin{array}{r}2020 \\
2020 \\
2009\end{array}$ \\
\hline Saúde mental e a pandemia COVID-19 & $\begin{array}{l}\text { WHO; } \\
\text { DE MORAES, et al.; } \\
\text { YAO et al.; } \\
\text { HAO et al.; } \\
\text { KOZLOFF et al.; } \\
\text { FERNÁNDEZ- } \\
\text { ARANDA et al.; } \\
\text { CARR. }\end{array}$ & \begin{tabular}{|l|}
2020 \\
2020 \\
2020 \\
2020 \\
2020 \\
2020 \\
2020
\end{tabular} \\
\hline Psicopatologias relacionadas ao isolamento social & $\begin{array}{l}\text { MARTINS et al.; } \\
\text { BROOKS et al.; } \\
\text { RUBIN et al.; } \\
\text { NERIA et al.; } \\
\text { LIM et al.; }\end{array}$ & $\begin{array}{l}2020 \\
2020 \\
2020 \\
2011 \\
2018\end{array}$ \\
\hline
\end{tabular}




\begin{tabular}{|c|c|c|}
\hline & HOLMES et al.. & 2020 \\
\hline $\begin{array}{l}\text { Estratégias para o acompanhamento de } \\
\text { psicopatologias oriundas do isolamento social }\end{array}$ & $\begin{array}{l}\text { BONANNO; } \\
\text { BONANNO et al.; } \\
\text { BLACK; LOBO; } \\
\text { BONANNO, et al.; }\end{array}$ & $\begin{array}{r}2004 \\
2008 \\
2008 \\
2015\end{array}$ \\
\hline Impactos da COVID-19 no Brasil & $\begin{array}{l}\text { AQUINO et al.; } \\
\text { GANEN et al.. }\end{array}$ & $\begin{array}{l}2020 \\
2020\end{array}$ \\
\hline
\end{tabular}

Fonte: Autores

Houve autores que foram aproveitados em mais de um tema.

A seguir serão apresentados os principais efeitos observados da Covid-19 na saúde mental por meio do levantamento dos efeitos psicológicos do isolamento social, a relação da saúde mental com a COVID-19, as principais psicopatologias observadas no isolamento social e as estratégias utilizadas no acompanhamento dessas psicopatologias.

\subsection{EFEITOS PSICOLÓGICOS DO ISOLAMENTO SOCIAL}

Brooks et al., (2020) realizaram uma revisão da literatura recente acerca dos efeitos psicológicos do isolamento social em participantes de dez países que foram submetidos ao isolamento por terem sido expostos ao Ebola, Influenza H1N1, SARS (Síndrome Respiratória Aguda Grave) e MERS (Síndrome Respiratória do Oriente Médio), dentre outros vírus de epidemias/pandemias anteriores, nos quais foram observados alguns fatores preditivos de sofrimento psicológico.

Indicadores sobre o demonstrar histórico de transtorno psiquiátrico prévio e o ser profissional de saúde, por exemplo, anteviram emoções negativas duradouras. Os profissionais de saúde, além de demonstrarem uma variedade de emoções negativas após o isolamento social (tristeza, nervosismo, culpa), apontaram sofrer maior estigma 
em comparação a outros profissionais. Esses dados apontam maior disponibilidade a sofrimento mental e, por consequência, maior necessidade de intervenção.

Outros fatores observados como antecessores de maior sofrimento mental foram a duração do período de isolamento social por mais de dez dias, medo de ser infectado ou infectar outras pessoas, frustração, tédio, falta de suprimentos e informações inadequadas sobre a doença (Brooks et al., 2020). Alguns desses indicadores foram atrelados a um maior número de sintomas de Transtorno de Estresse Pós-Traumático (TEPT), transtornos de ansiedade e afetos negativos após o final do isolamento social. Desta forma, as perdas financeiras e as estigmas, bem como outras condições adversas ocasionadas pelo isolamento social, foram identificadas como fatores de risco quanto ao desenvolvimento de transtornos mentais e de afetos negativos prolongados.

Identificou-se também que os prejuízos financeiros, apresentam-se como o maior fator de risco no desenvolvimento de transtornos mentais ligados a famílias de nível socioeconômico baixo, em razão da representação dessa renda a essas famílias e do impacto da perda da mesma (Brooks et al., 2020). Evidências apontam que 30\% da amostra de alguns estudos a respeito dos participantes submetidos ao isolamento social apresentavam critérios para o diagnóstico de TEPT (ZANON et al., 2020).

A prevalência contínua de sintomas de trauma em indivíduos que foram expostos a pandemias, sujeitando-se aos períodos de isolamento social, pode ser possivelmente explicada pelo fato do medo e da percepção de risco trabalharem como amplificadores de informações pouco claras, nas quais são comuns nos períodos iniciais de surtos (JOHAL, 2009). Assim como o desconhecimento da doença e a ausência de vacinas e remédios representam outros fatores relevantes. Neste sentido, a cobertura de pandemias por fontes de notícias autênticas compõe um fator desencadeante de medo, ao passo que noticiam algo importante e, talvez, a existência de uma possível ameaça a população, difícil de controlar. 


\subsection{SAÚDE MENTAL E A PANDEMIA COVID-19}

Emergências de saúde pública podem afetar a saúde, segurança e bem-estar de indivíduos e comunidades por causa de perdas econômicas, fechamentos de postos de trabalho e escolas, emprego inadequado de recursos para assistência médica e distribuição deficiente das necessidades. Esses efeitos podem se traduzir em uma série de reações emocionais percebidas através de sofrimento ou condições psiquiátricas, comportamentos não saudáveis como uso excessivo de substâncias e não conformidade com as diretrizes de saúde pública (como confinamento domiciliar e vacinação) em pessoas que contraem a doença, e na população em geral. Pesquisas em saúde mental no âmbito de desastres estabeleceram que o sofrimento emocional é frequente nas populações afetadas - uma descoberta que certamente terá eco nas populações afetadas pela pandemia de Covid-19 (WHO, 2020a).

A pandemia Covid-19 tem implicações alarmantes para a saúde individual e coletiva e para o funcionamento emocional e social. Os provedores de assistência médica, já sobrecarregados, além de prestarem serviços de assistência médica, têm um papel importante no monitoramento das necessidades psicossociais e no fornecimento de apoio psicossocial a seus pacientes, profissionais de saúde e ao público - atividades que devem ser integradas à assistência médica geral para pandemia (DE MORAES et al., 2020).

Pessoas com transtornos mentais pré-existentes relataram aumento dos sintomas e pior acesso a serviços e suporte desde o início da pandemia COVID-19 (YAO et al., 2020). A alta antecipada de unidades psiquiátricas e a interrupção do atendimento psiquiátrico face a face tornaram-se comuns, apresentando consequências negativas dos quais podem incluir recaídas, comportamento suicida, falta de acesso a cuidados médicos e isolamento social. Quarentena e bloqueio podem afetar particularmente pessoas com problemas de saúde mental pré-existentes: aumento de sintomas de ansiedade e depressão e altas taxas de transtorno de estresse pós-traumático e insônia foram relatados. (HAO et al., 2020). 
Simultaneamente, o distanciamento físico reduziu a disponibilidade de muitos apoios familiares, sociais e psiquiátricos. Pessoas com doenças mentais graves e desvantagens socioeconômicas associadas estão particularmente sob risco dos efeitos diretos e indiretos da pandemia. (KOZLOFF et al., 2020). Da mesma forma, sintomas aumentados e vulnerabilidade foram relatados durante a pandemia de COVID-19 em pessoas com transtornos alimentares, transtorno do espectro do autismo, demência e deficiências intelectuais e de desenvolvimento. (FERNÁNDEZARANDA et al., 2020). O confinamento em casa, a interrupção da rotina diária e o distanciamento físico podem agravar todas essas condições e representar um desafio para os usuários e cuidadores dos serviços.

Muitos países têm equipes dedicadas para fornecer apoio em saúde mental para profissionais de saúde e serviços de ligação psiquiátrica. O apoio necessário depende do estágio da pandemia. Muitas questões permanecem sobre como mitigar os efeitos da pandemia COVID-19 na saúde mental. Os profissionais de saúde mental com experiência em ciências sociais e serviços comunitários também devem aconselhar os reguladores a desenvolver, implementar e avaliar estratégias para lidar com a pandemia e seus desdobramentos.

Para pessoas em sofrimento agudo que correm o risco de desenvolver doenças de longo prazo e aquelas que não confiam ou não se envolvem com os serviços de saúde mental convencionais, a facilitação do acesso diversificado e flexível aos cuidados de saúde mental é particularmente importante. Organizações lideradas por comunidades, usuários e famílias locais e pequenas iniciativas, independentes de apoio a pares, se mobilizaram rapidamente para fornecer ajuda e orientação imediatas durante a pandemia. Esses serviços de apoio à comunidade responderam proativamente ao COVID-19 de maneira diferente dos serviços clínicos convencionais e poderiam se expandir de maneira econômica para dar suporte a um aumento esperado na demanda por serviços (CARR, 2020). Entretanto, eles podem não ser apropriados ou suficientes para todos e, portanto, devem complementar, mas não substituir, os cuidados de saúde mental convencionais. 


\subsection{PSICOPATOLOGIAS RELACIONADAS AO ISOLAMENTO SOCIAL}

Muitas das notícias publicadas no COVID-19 são angustiantes e, às vezes, as notícias estão associadas a boatos, razão pela qual os níveis de ansiedade aumentam quando uma pessoa é constantemente exposta às notícias do COVID-19. A desinformação e os relatórios fabricados sobre o COVID-19 podem exacerbar os sintomas depressivos na população em geral.

Pesquisas emergentes sobre os efeitos psicológicos do COVID-19 têm focado principalmente na compreensão de medo excessivo ou reações de ansiedade. Um fenômeno igualmente importante, mas negligenciado, diz respeito a ausência de medo e ansiedade frente ao COVID-19; de fato, pessoas com baixo nível de ansiedade sobre um surto infeccioso têm menos propensão a aderir às recomendações de saúde pública (por exemplo, distância social).

Nos países subdesenvolvidos e em desenvolvimento, que inclui o Brasil, as condições epidêmicas da COVID-19 impõem maiores efeitos psicológicos à população, visto que esses países também são afetados por muitas outras doenças infecciosas como as doenças endêmicas que são transmitidas por vetores e que até hoje ainda tem alta incidência nestas populações. A incerteza sobre o estado de saúde, o acompanhamento dos pacientes, os cuidados com o tratamento e a ineficiência nessas comunidades também podem aumentar a vulnerabilidade dessas comunidades aos efeitos psicológicos do COVID-19 (MARTINS et al., 2020).

O nervosismo e a ansiedade em uma sociedade afetam a todos em grande medida. Evidências recentes sugerem que as pessoas que são mantidas em isolamento e quarentena experimentam níveis significativos de ansiedade, raiva, confusão e estresse (BROOKS et al., 2020). Em geral, todos os estudos que examinaram os distúrbios psicológicos durante a pandemia COVID-19 relataram que os indivíduos afetados apresentam vários sintomas de trauma mental, como sofrimento emocional, depressão, estresse, alterações de humor, irritabilidade, insônia, déficit de atenção, transtorno de hiperatividade, estresse pós-traumático e raiva (RUBIN et al., 2020). 
A pesquisa também mostrou que a exposição frequente à mídia pode causar angústia (NERIA et al., 2011). No entanto, na situação atual, é desafiador prever com precisão as consequências psicológicas e emocionais do COVID-19. Os resultados de estudos epidemiológicos mostram que as mulheres correm um risco maior de depressão. As mulheres são mais vulneráveis ao estresse e transtorno de estresse pós-traumático do que os homens (LIM et al., 2018).

Estudos recentes revelaram uma associação entre o histórico médico e o aumento da ansiedade e da depressão causada pela propagação do COVID-19. Trabalhos de pesquisa anteriores mostraram que a história médica e as doenças crônicas estão associadas a níveis aumentados de sofrimento psiquiátrico. Pessoas com histórico de problemas médicos e com problemas de saúde podem se sentir mais vulneráveis a uma nova doença (HOLMES et al., 2020).

\subsection{ESTRATÉGIAS PARA O ACOMPANHAMENTO DE PSICOPATOLOGIAS ORIUNDAS DO ISOLAMENTO SOCIAL}

A principal tarefa psicológica para a maioria das pessoas durante a pandemia é manter o sofrimento ao mínimo. No entanto, em face da incerteza causada pela pandemia, as perguntas não faltam. Quão comum será a resiliência? O que os indivíduos podem fazer em face da pandemia COVID-19 para manter a resiliência? Os estudos específicos para COVID-19 que podem responder a essas perguntas estão apenas começando. No entanto, pesquisas anteriores relacionadas a surtos de doenças e desastres naturais forneceram informações importantes que podem fornecer diretrizes para nos ajudar a superar a pandemia. Neste comentário, abordamos essas questões importantes e sugerimos algumas direções de pesquisa importantes.

Estudos sobre a exposição ao estresse extremo, como surtos de doenças, relataram uma prevalência mais alta de psicopatologia e uma taxa mais baixa de resiliência em comparação com estudos que examinaram eventos apenas moderadamente aversivos. Em todos os tipos de estudos e abordagens de modelagem, a proporção de indivíduos resilientes foi, em média, cerca de dois terços (BONANNO, 2004). 
Para aqueles infectados com COVID-19, pode ser especialmente importante mobilizar apoio social, aumentar comportamentos de busca de ajuda e manutenção de cuidados físicos contínuos para manter a resiliência (BONANNO et al., 2008).

No nível familiar, estudos identificaram alguns fatores de resiliência, como adaptabilidade, coesão familiar, boa comunicação e gestão financeira adequada (BLACK; LOBO, 2008). Embora esses estudos contribuam para a nossa compreensão das trajetórias individuais, poucos estudos examinaram especificamente os resultados resilientes no nível familiar (BONANNO et al., 2015). Dado que muitos indivíduos são colocados em quarentena com membros da família, é importante considerar caminhos para a resiliência no nível familiar.

No nível da comunidade, resultados resilientes são mais prováveis de ocorrer em comunidades com maior coesão social, menores taxas de crime e menos exposição a desastres. Outros fatores importantes incluem o possível impacto negativo das disparidades de saúde, como acesso a cuidados de saúde e status socioeconômico no medo e na ansiedade (BONANNO et al., 2015). Pesquisas destinadas a compreender melhor as origens das disparidades de saúde no contexto do COVID19, os efeitos da desigualdade em saúde percebida na psicopatologia e as políticas associadas que se esforçam para reduzir a lacuna de desigualdade são cruciais para manter a resiliência da comunidade.

\subsection{IMPACTOS DA COVID-19 NO BRASIL}

O Brasil registrou o primeiro caso da América Latina em fevereiro de 2020, após a confirmação laboratorial da COVID-19, o paciente recebeu os cuidados padronizados pela vigilância epidemiológica, mantendo-se em isolamento domiciliar, ao passo que os familiares que tiveram contato com o sujeito, no hospital onde foi atendido e no voo de retorno da Itália, eram investigados. Daí por diante, a epidemia tem se alastrado no país. É importante ressaltar que como o Brasil não adotou estratégias amplas de testagem na população, há grande possibilidade de subnotificações no número de casos e óbitos (AQUINO et al., 2020). 
Visto que o avanço tecnológico ocorre em função de um avanço nas políticas e nas práticas humanas e sociais, compreende-se que a saúde mental é essencial para a manutenção das capacidades de criatividade e produtividade do ser humano. Desta forma, o estudo se justifica pela necessidade de melhor direcionar e balizar as campanhas e as propostas de controle sobre a disseminação do COVID-19, visto que os níveis de saúde mental da população se refletem no comportamento dos cidadãos, que podem acatar mais ou menos às políticas de distanciamento.

Ainda que a Lei ํㅜ 13.979/2020, que aborda sobre as medidas para enfrentamento da COVID-19 no Brasil, vigore desde 7 de fevereiro, isto é, desde o momento anterior ao início oficial da epidemia, o presidente da república tem subestimado sua relevância, mantendo-se como um dos poucos líderes mundiais que se negam a ameaça que ela constitui. Diversas são as matérias jornalísticas comunicando o seu posicionamento opositor às medidas adotadas nos Estados e municípios e o incentivo aos seus adeptos nas redes sociais ao descumprimento das recomendações de distanciamento social. Um impasse político claro se instaurou entre o presidente e o então Ministro da Saúde, que defendia as medidas orientadas pela OMS e apoiava as iniciativas locais e regionais mais rigorosas de medidas de controle da COVID-19 (AQUINO et al., 2020).

Inseridos nesse cenário político, acrescido à crise sanitária, uma importante crise política, a implantação das medidas de controle, que incluem o distanciamento social, tem sido garantida pelos governadores e prefeitos, em especial nos estados mais afetados. A autonomia administrativa dos estados e dos municípios no âmbito da saúde, da educação e do comércio, prevista na Constituição Federal, diminui a ocorrência da interferência direta do governo federal em decisões de governos locais. Sendo isso, objeto de discussão pelo Supremo Tribunal Federal. Contudo, até então, o reconhecimento da autonomia dos estados e dos municípios sobre à adoção de medidas de emergência relacionadas à saúde pública tem prevalecido.

Grande parte dos Estados e o Distrito Federal adotaram práticas e medidas para minimizar a circulação e a aglomeração de pessoas em maior ou menor grau. Contudo, o Governo Federal, ao subestimar a relevância do distanciamento social e 
opor-se às medidas adotadas pelos Estados e municípios teve prejuízo na adesão da população a elas. Neste sentido, um estudo brasileiro aplicou uma modelagem matemática para estimar o efeito das medidas de distanciamento social sobre a Região Metropolitana de São Paulo (AQUINO et al., 2020).

Este estudo evidenciou que, se as medidas de distanciamento social não fossem adotas, a capacidade dos UTIs para o tratamento da COVID-19 seria superada no primeiro mês em cerca de $130 \%$, ao passo que no segundo mês isso seria correspondente a 14 vezes. Sugerindo também que "o conjunto das medidas de distanciamento social implementados (e sua manutenção no presente momento) poderá evitar a sobrecarga do sistema de saúde (mantendo a ocupação em 76\%) e a morte de quase 90 mil pessoas ao longo da epidemia" (AQUINO et. al., 2020 p. 2429). Em adicional, o estudo indica o uso de dados de hospitalização de SRAG (Síndromes Respiratórias Agudas Graves) para o acompanhamento do impacto das medidas de distanciamento social (GANEN et al., 2020).

De acordo com as notícias mais recentes, a epidemia da COVID-19 no Brasil ainda está na primeira fase, e a crise política, alimentada pela substituição do Ministro da Saúde, adiciona mais inseguranças no tocante às políticas que poderão ser adotadas pelo Governo Federal. Os levantamentos científicos sugerem que a junção de isolamento dos casos, quarentena de contatos e medidas ampliadas de distanciamento social, em especial aquelas que diminuem em pelo menos $60 \%$ os contatos sociais, têm o potencial de minimizar a transmissão da doença. Ainda que pouco explorada na literatura sobre o tema no contexto brasileiro, a observação anterior de países asiáticos e europeus sugere que as estratégias de distanciamento social devem ser estimuladas e aplicadas e coordenadas entre as diferentes esferas governamentais e regiões objetivando alcançar o fim da epidemia o mais brevemente possível, bem como para evitar novas ondas do contágio da doença.

Sua implementação na realidade brasileira é sem dúvida um grande desafio. A desigualdade social do país, com muitas pessoas em situação de pobreza e a parte crescente de pessoas vivendo nas ruas, somados ao grande número de indivíduos privados de liberdade, podem contribuir com a transmissão e dificultar a 
implementação do distanciamento social. Em adicional, a importante parcela dos trabalhadores informais requerem que, para garantir a sustentabilidade e a real efetividade das medidas de controle da COVID-19, sejam aplicadas políticas de proteção social e suporte a indivíduos em situação vulnerável. Políticas como a renda mínima que certificam a proteção ao trabalho dos trabalhadores que têm vínculos laborais formais são imprescindíveis para garantir a sobrevivência das pessoas, não somente enquanto perdurarem as restrições para o desenvolvimento das atividades econômicas.

Mesmo que o isolamento social seja considerado como uma fonte de ansiedade e estresse a população, essa variável não é apontada significativamente no modelo de regressão. Tais levantamentos podem sugerir que o distanciamento social e a restrição do contato físico entre as pessoas durante a pandemia não são, intrinsecamente, um fator de risco para o adoecimento mental; mas sim que outros fatores que permeiam esse contexto influenciam o desenvolvimento de tal doença. Os prejuízos financeiros em razão dos reflexos da doença sobre o cenário econômico local e, ainda, a exposição a informações negativas sobre a COVID-19 são fatores que propiciam riscos à saúde mental. Exigindo, deste modo, uma atenção especial quanto aos fatores econômicos e a perda na renda familiar, de modo a reforçar a necessidade de políticas públicas e benefícios de auxílio financeiro durante este período.

Assim, como o indicado pela Organização Mundial da Saúde, verifica-se que os transtornos mentais e comportamentais representam uma das principais causas de afastamento do trabalho. Em virtude disso, identifica-se que a propiciação de bons índices de saúde mental auxilia o comportamento preventivo e a manutenção da saúde da população, possibilitando que estas pessoas estejam em condições de retorno às suas atividades, logo após a pandemia, sendo isso fundamental a recuperação da economia do país. 


\section{CONCLUSÃO}

O COVID-19 não só causa problemas de saúde física, mas também resulta em uma série de distúrbios psicológicos. A disseminação do novo Coronavírus pode impactar a saúde mental de pessoas em diferentes comunidades. Assim, é essencial preservar a saúde mental dos indivíduos e desenvolver intervenções psicológicas que possam melhorar a saúde mental de grupos vulneráveis durante a pandemia de COVID-19. Portanto, pode-se concluir que a pandemia de COVID-19 pode afetar a saúde mental de indivíduos e diferentes comunidades.

$\mathrm{Na}$ crise atual, é imprescindível identificar indivíduos sujeitos a transtornos psicológicos em diferentes grupos e em diferentes camadas da população, para que, com estratégias psicológicas, técnicas e intervenções adequadas, a saúde mental da população em geral seja preservada e melhorada. O que a maioria está sentindo são os efeitos de perdas transitórias.

As implicações econômicas da pandemia COVID-19 são sérias. É importante estar ciente dos riscos de promover soluções baratas para ampliar o acesso aos cuidados de saúde mental. Os cuidados de saúde mental de baixa qualidade com base na acessibilidade sem avaliação da qualidade ou monitoramento das necessidades e da eficiência só contribuirão para aumentar as desigualdades e piorar a saúde mental.

\section{REFERÊNCIAS}

AQUINO, Estela ML et al. Medidas de distanciamento social no controle da pandemia de COVID-19: potenciais impactos e desafios no Brasil. Ciência \& Saúde Coletiva, v. 25, p. 2423-2446, 2020.

BEZERRA, Anselmo César Vasconcelos et al. Fatores associados ao comportamento da população durante 0 isolamento social na pandemia de CoVID-19. Ciência \& Saúde Coletiva, v. 25, p. 2411-2421, 2020.

BLACK, Keri; LOBO, Marie. Uma revisão conceitual dos fatores de resiliência familiar. Journal of family enfermagem, v. 14, n. 1, pág. 33-55, 2008. 
BONANNO, George A. Perda, trauma e resiliência humana: subestimamos a capacidade humana de prosperar após eventos extremamente aversivos? Psicólogo Americano, v. 59, n. 1, pág. 20, 2004.

BONANNO, George A.; MANCINI, Anthony D. A capacidade humana de prosperar diante de um potencial trauma. Pediatrics, v. 121, n. 2, pág. 369-375, 2008.

BONANNO, George A.; ROMERO, Sara A.; KLEIN, Sarah I. Os elementos temporais da resiliência psicológica: uma estrutura integrativa para o estudo de indivíduos, famílias e comunidades. Investigação Psicológica, v. 26, n. 2, pág. 139169, 2015.

BROOKS, Samantha K. et al. El impacto psicológico de la cuarentena y cómo reducirla: revisión rápida de las pruebas. Lancet, v. 395, p. 912-20, 2020.

CARR, Sarah. Assistência Social para Comunidades Marginalizadas: Equilibrando auto-organização, micro-provisão e apoio geral. Documento de política, v. 18, 2014.

DA SILVA, Carlos Eduardo Menezes et al. Influência das condições de bem-estar domiciliar na prática do isolamento social durante a Pandemia da Covid19. Journal of Health \& Biological Sciences, v. 8, n. 1, p. 1-7, 2020.

DE MORAES, Érica Brandão et al. A segurança dos profissionais de saúde em tempos de COVID-19: uma reflexão. Research, Society and Development, v. 9, n. 7, p. e134973832-e134973832, 2020.

DE SOUSA CARVALHO, Leilanir et al. O impacto do isolamento social na vida das pessoas no período da pandemia da COVID-19. Research, Society and Development, v. 9, n. 7, p. e998975273-e998975273, 2020.

DUARTE, Michael de Quadros et al. COVID-19 e os impactos na saúde mental: uma amostra do Rio Grande do Sul, Brasil. Ciência \& Saúde Coletiva, v. 25, p. 3401-3411, 2020. 
FERNÁNDEZ-ARANDA, Fernando et al. COVID - 19 e implicações para transtornos alimentares. European Eating Disorders Review, v. 28, n. 3, pág. 239, 2020.

GANEM, Fabiana et al. The impact of early social distancing at COVID-19 Outbreak in the largest Metropolitan Area of Brazil. MedRxiv, 2020.

$\mathrm{HAO}$, Fengyi et al. Os pacientes psiquiátricos apresentam mais sintomas psiquiátricos durante a pandemia e bloqueio de COVID-19? Um estudo de casocontrole com implicações de serviço e pesquisa para imunopsiquiatria. Brain, Behavior, and Immunity, 2020.

HOLMES, Emily A. et al. Prioridades de pesquisa multidisciplinar para a pandemia COVID-19: um chamado para ação para as ciências da saúde mental. The Lancet Psychiatry, 2020.

HOSSAIN, Md Mahbub; SULTANA, Abida; PUROHIT, Neetu. Resultados de saúde mental da quarentena e isolamento para prevenção de infecções: uma revisão sistemática abrangente das evidências globais. Disponível em SSRN 3561265, 2020.

JOHAL, Sarbjit S. Impactos psicossociais da quarentena durante surtos de doenças e intervenções que podem ajudar a aliviar a tensão. 2009.

JUNIOR, Rodolpho Telarolli. Epidemias no Brasil: uma abordagem biológica e social. 3. ed. São Paulo: Moderna, 2012.

KOZLOFF, Nicole et al. A pandemia global COVID-19: implicações para pessoas com esquizofrenia e transtornos relacionados. Boletim da Esquizofrenia, 2020.

KRAEMER, Moritz UG et al. O efeito da mobilidade humana e das medidas de controle na epidemia de COVID-19 na China. Science, v. 368, n. 6490, pág. 493-497, 2020.

LIM, Grace Y. et al. Prevalência de depressão na comunidade de $\mathbf{3 0}$ países entre 1994 e 2014. Relatórios Científicos, v. 8, n. 1, pág. 1-10, 2018. 
MARTINS, MARCELI TOMÉ; MARTINI, SANDRA REGINA. Covid-19 na perspectiva dos países fundadores do Mercosul: uma análise dos cenários a partir dos discursos presidenciais e consequentes medidas tomadas pelos líderes1. O Direito à Saúde Frente à Pandemia COVID-19: da crise sanitária à crise.

MAVROUDEAS, Stavros D. 3. A pandemia de coronavírus e a crise econômica e da saúde1. Covid-19, Capitalismo e Crise: bibliografia comentada, p. 113, 2020.

NERIA, Yuval; SULLIVAN, Gregory M. Compreendendo os efeitos na saúde mental da exposição indireta ao trauma em massa por meio da mídia. Jama, v. 306, n. 12, pág. 1374-1375, 2011.

ORNELL, FELIPE et al. Pandemia de medo e COVID-19: impacto na saúde mental e possíveis estratégicas. Revista debates in psychiatry, 2020.

PEREIRA, Mara Dantas et al. A pandemia de COVID-19, o isolamento social, consequências na saúde mental e estratégias de enfrentamento: uma revisão integrativa. Research, Society and Development, v. 9, n. 7, p. e652974548e652974548, 2020.

REIS-FILHO, José Amorim et al. COVID-19, Afastamento social, Pesca artesanal e Segurança alimentar: Como esses temas estão relacionados e quão importante é a soberania dos trabalhadores da pesca diante do cenário distópico. 2020.

RIBEIRO, Eliane Gusmão et al. Saúde Mental na Perspectiva do Enfrentamento à COVID-19: Manejo das Consequências Relacionadas ao Isolamento Social. Revista Enfermagem e Saúde Coletiva-REVESC, v. 5, n. 1, p. 47-57, 2020.

RUBIN, G. James; WESSELY, Simon. Os efeitos psicológicos de colocar uma cidade em quarentena. Bmj, v. 368, 2020.

SHANAHAN, Lilly et al. Sofrimento emocional em adultos jovens durante a pandemia COVID-19: evidências de risco e resiliência em um estudo de coorte longitudinal. Medicina psicológica, p. 1-10, 2020. 
SHARMA, Arpit. Finding community during a pandemic. Science (New York, NY), v. 368, n. 6487, p. 206, 2020.

SCHMIDT, Beatriz et al. Impactos na Saúde Mental e Intervenções Psicológicas Diante da Pandemia do Novo Coronavírus (COVID-19). 2020.

TEIXEIRA, Sandra. Novo Coronavírus (Covid-19): informações básicas. Ministério da Saúde, Rede Bibliosus, PÁGINA INICIAL, ARTIGOS, NOTÍCIAS, NOVO CORONAVÍRUS (COVID-19): INFORMAÇÕES BÁSICAS. Publicado 09 de Março de 2020, 17h51. Disponível em: $<$ http://bibliosus.saude.gov.br/index.php/artigos/14-noticias/244-novo-coronaviruscovid-19-informacoesbasicas\#: :text=A\%20Covid\%2D19\%20\%C3\%A9\%20uma,algu\%C3\%A9m\%20doen te\%20tosse\%20ou\%20espirra>

WHO, World Health Organization. (2020a). Mental health and psychosocial considerations during the COVID-19 outbreak. Disponivel em: $<$ https://www.who.int/docs/default-source/coronaviruse/situation-reports/20200514covid-19-sitrep-115.pdf?sfvrsn=3fce8d3c_6>

WHO, World Health Organization. (2020b). (COVID-19) situation reports - 115. Disponível em: <https://www.who.int/docs/default-source/coronaviruse/situationreports/20200514-covid19-sitrep-115.pdf?sfvrsn=3fce8d3c_6>

YAO, Hao; CHEN, Jian-Hua; XU, Yi-Feng. Pacientes com transtornos mentais na epidemia de COVID-19. The Lancet Psychiatry, v. 7, n. 4, pág. e21, 2020.

ZANON, Cristian et al. COVID-19: implicações e aplicações da Psicologia Positiva em tempos de pandemia. Estudos de Psicologia (Campinas), v. 37, 2020.

Enviado: Dezembro, 2020.

Aprovado: Janeiro, 2021. 